\title{
SPONTANEOUS IMBIBITION PROCESS IN MICRO-NANO FRACTAL CAPILLARIES CONSIDERING SLIP FLOW
}

\author{
YINGHAO SHEN, ${ }^{*, \dagger}$ CAOXIONG LI, ${ }^{*, \dagger, \ddagger, \|}$ HONGKUI GE, $,{ }^{*}, \S$ XUEJING GUO \\ and SHAOJUN WANG \\ *Unconventional Natural Gas Institute, China University of Petroleum \\ Beijing 102249, P. R. China \\ †State Key Laboratory of Petroleum Resources and Prospecting \\ Beijing 102249, P. R. China \\ ${ }^{\ddagger}$ School of Engineering Science, University of Chinese Academy of Sciences \\ Beijing 100049, P. R. China \\ ${ }^{\S}$ China University of Petroleum-Beijing at Karamay \\ Karamay 834000, P. R. China \\ ${ }^{\top}$ CNPC Research Institute of Petroleum Exploration \\ and Development, Beijing 10083, P. R. China \\ Ilicaoxiong@imech.ac.cn
}

Received September 4, 2017

Accepted December 4, 2017

Published February 26, 2018

\begin{abstract}
An imbibition process of water into a matrix is required to investigate the influences of large-volume fracturing fluids on gas production of unconventional formations. Slip flow has been recognized by recent studies as a major mechanism of fluid transport in nanotubes. For nanopores in shale, a slip boundary is nonnegligible in the imbibition process. In this study, we established an analytic equation of spontaneous imbibition considering slip effects in capillaries. A spontaneous imbibition model that couples the analytic equation considering the slip effect was constructed based on fractal theory. We then used a model for various conditions,
\end{abstract}

\footnotetext{
"Corresponding author.
}

This is an Open Access article published by World Scientific Publishing Company. It is distributed under the terms of the Creative Commons Attribution 4.0 (CC-BY) License. Further distribution of this work is permitted, provided the original work is properly cited. 
such as slip boundary, pore structure, and fractal dimension of pore tortuosity, to capture the imbibition characteristics considering the slip effect. A dynamic contact angle was integrated into the modeling. Results of our study verify that the slip boundary influences water imbibition significantly. The imbibition speed is significantly improved when slip length reaches the equivalent diameter of a tube. Therefore, disregarding the slip effect will underestimate the imbibition speed in shale samples.

Keywords: Spontaneous Imbibition; Fractal; Slip Flow; Nanopores; Capillary Pressure.

\section{INTRODUCTION}

Unconventional formation affects oil and gas supplies. ${ }^{112}$ Large-scale hydraulic fracturing is a key technology for successfully developing unconventional formation $\frac{3}{3}$ Thousands of tons of liquid are pumped into the formation, and the injected liquid significantly influences fracturing network, $\frac{4-6}{6}$ formation damage, $\frac{78}{7}$ mechanical behavior ${ }^{9}$ and production rate post fracturing. 10111 The mechanism of water transport into formation matrix and modeling remains difficult to explain in unconventional formation, although the interaction between fracturing fluids and formation has been extensively studied $\frac{1213}{10}$ in recent years given the specific characteristics of unconventional formation, such as ultralow permeability, strong heterogeneity, and complex mineral composition! 14 17

Spontaneous imbibition is a dominant mechanism of water transport into formation considering the high capillary pressure in nanopores 18$] 21$ A research development of spontaneous imbibition is summarized systematically, and the main models include Lucas-Washburn, Terzaghi, Handy, Mattax and Kyte, and Aronofsky ${ }^{222}$ Fractal theory is powerful in studying the imbibition in complex porous media. 23] 25$]$ Fractal structure parameters are considered to explore the spontaneous imbibition into a fractal tree-like network, $\frac{26}{26}$ and the influences of tortuosity, asymmetry, and wettability on capillary rise are investigated based on fractal theory ${ }^{[27}$ Also, fractal theory is helpful for permeability evaluation in rock fracture networks. 2829

The nanopores of unconventional formation cause the transport mechanism to be significantly different from the conventional formation. $30+32$ Javadpour et al! $!^{33}$ studied gas flow in nanoscale and highlighted the special flow regime of nanopores. Slip flow is considered a major mechanism of gas transport in nanotubes, and a related model is proposed 134 A recent study has identified significant differences in no-slip boundary condition in nanotubes, $\frac{35136}{36}$ and conventional flow equations, such as Darcy's law, may not be valid for shale systems given the difference in the controlling physics of liquid flow. A pore network simulation considering pore geometry and slip effect has proven that slip flow plays an important role in fluid flow in the porous media of shale given the slip effect for pore network simulation.

\section{SPONTANEOUS IMBIBITION MODEL}

\subsection{Basic Assumptions}

This study aims to establish a preliminary understanding of the effect of slip boundary on water imbibition in shale by using a fractal approach. We formulated an analytic equation of spontaneous imbibition considering a slip effect in a single capillary. A spontaneous imbibition model in porous media is proposed based on the fractal characteristics of the porous media and coupling the analytic equation. The influences of slip boundary, fractal dimension, and pore tortuosity are investigated according to the model.

Several assumptions are generated in the model. First, liquid is the wetting phase, and gas is the nonwetting phase. Second, the liquid is a Newton liquid with laminar flow and without inertial forces. Third, the driving force of spontaneous imbibition is a capillary force. Fourth, slip occurs in the tube wall. Fifth, Liu et al ${ }^{[37}$ assumed that each capillary cuts through the model.

\subsection{Analytic Equation of Spontaneous Imbibition Considering a Slip Effect in a Single Capillary}

We derive the analytic equation of spontaneous imbibition in this section. The effect of liquid slip is considered. The liquid slip effect can significantly increase liquid flux in nanopores. Figure 1 illustrates a thin layer of liquid formed on the wall of 


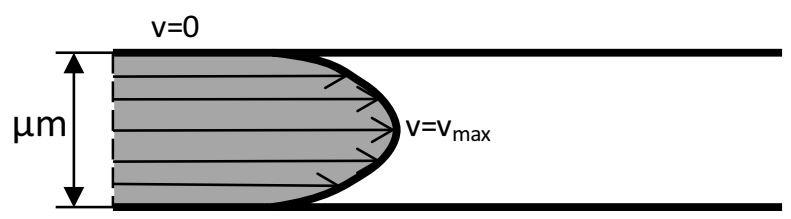

(a)

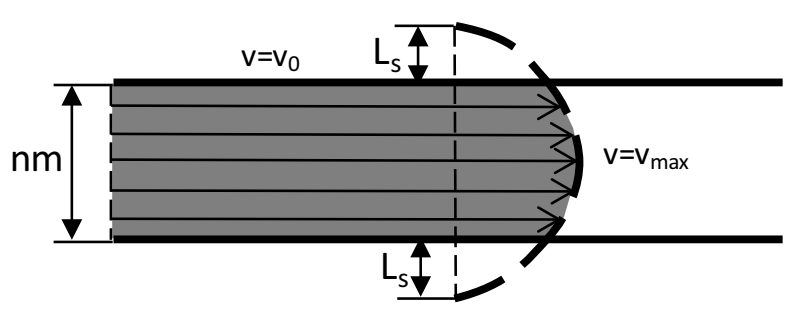

(b)

Fig. 1 Schematic of slip condition in micro- (a) and nanoscale (b) systems!

nanopores, thereby leading to a liquid slip on the wall, that is, the slip effect. The velocity distribution in the cross-section follows a parabolic curve, and a slip length $L_{s}$ is assumed to exist.

The fluid flux, which considers liquid slip, is expressed as

$$
Q=\frac{\pi \Delta p}{2 \mu L}\left[\frac{1}{2}\left(R+L_{s}\right)^{2} R^{2}-\frac{1}{4} R^{4}\right],
$$

where $Q$ is the fluid flux in the tube, $\Delta p$ is the pressure difference in the fluid, $\mu$ is the dynamic viscosity, $L$ is the length of fluid pathline, $R$ is the equivalent radius of the tube, and $L_{s}$ is the slip length. A hydraulic diameter is introduced in noncircular tubes:

$$
D_{h}=\frac{4 A_{c}}{C},
$$

where $D_{h}$ is the hydraulic diameter, $A_{c}$ is the crosssectional area, and $C$ is the wetted perimeter.

The liquid flux $Q$ in the noncircular tubes can be expressed as

$$
Q=\alpha^{4} \frac{\pi \Delta p}{32 \mu L}\left[\frac{1}{2}\left(D_{h}+2 L_{s}\right)^{2} D_{h}^{2}-\frac{1}{4} D_{h}^{4}\right],
$$

where $\alpha$ is a geometry correction factor that depends on the capillary shape. Franken et al. calculated $\alpha$ for several shapes. $\alpha=1$ is the circular cross-section, $\alpha=1.186$ for an equilateral triangle, and $\alpha=1.094$ for a square. The values of $\alpha$ for other shapes are presented in Cai et al ${ }^{[38}$ The value of $\alpha$ represents circular geometry when this value approaches 1; a large value of $\alpha$ implies a significant deviation in a circular geometry, such as silts.
For a square cross-section, the liquid flux in tortuous capillaries can be expressed as

$$
Q=\alpha^{4} \frac{\pi \Delta p}{32 \mu L_{f}}\left[\frac{1}{2}\left(\lambda+2 L_{s}\right)^{2} \lambda^{2}-\frac{1}{4} \lambda^{4}\right],
$$

where $\lambda$ is the equivalent diameter or side length of a square, and $L_{f}$ is the length of fluid pathline for tortuous tubes. For straight tube, $L_{f}=L$.

For $\Delta p$, which represents the pressure difference between driving force and resistance, we can derive

$$
\Delta p=p_{c}-\rho g L_{0},
$$

where $L_{0}$ is the vertical height of meniscus. $L_{0}$ is the increase in time, and $p_{c}$ is the main driving force. According to Young-Laplace equation,

$$
p_{c}=\frac{2 \sigma \cos \theta}{r},
$$

where $\sigma$ is the interfacial tension between gas and liquid, $\theta$ is the contact angle, and $r$ is the tube radius. In noncircular tubes, $p_{c}$ is expressed as

$$
p_{c}=\frac{4 \sigma \cos \theta}{\alpha D_{h}}=\frac{4 \sigma \cos \theta}{\alpha \lambda} .
$$

According to the work of Hilpert, 39 several experimental and numerical studies ${ }^{40141}$ on capillary tube flows have shown that the contact angle $\theta$ in Young-Laplace equation should be a dynamic contact angle. Hilpert ${ }^{42}$ provided a linear function for a dynamic contact angle as

$$
\cos \theta-\cos \theta_{\mathrm{eq}}=\tilde{\alpha} \mathrm{Ca}=-\tilde{\alpha} \frac{d L_{f}}{d t} \frac{\mu}{\sigma},
$$

where $\theta_{\text {eq }}$ is the equilibrium contact angle, and $\tilde{\alpha} \geq 0$ is a nondimensional parameter. $\mathrm{Ca}$ is the capillary number. Therefore, the $p_{c}$ for the dynamic angle can be expressed as

$$
p_{c}=\frac{4 \sigma \cos \theta}{\alpha D_{h}}=\frac{4 \sigma}{\alpha \lambda}\left(\cos \theta_{\mathrm{eq}}-\tilde{\alpha} \frac{d L_{f}}{d t} \frac{\mu}{\sigma}\right) .
$$

The liquid flux $Q$ that considers slip flow, dynamic contact angle, and gravity can be expressed as

$$
\begin{aligned}
Q= & \alpha^{4} \frac{\pi}{32 \mu L_{f}}\left[\frac{1}{2}\left(\lambda+2 L_{s}\right)^{2} \lambda^{2}-\frac{1}{4} \lambda^{4}\right] \\
& \times\left[\frac{4 \sigma}{\alpha \lambda}\left(\cos \theta_{\mathrm{eq}}-\tilde{\alpha} \frac{d L_{f}}{d t} \frac{\mu}{\sigma}\right)-\rho g L_{0}\right] .
\end{aligned}
$$

The imbibition velocity is

$$
v_{f}=\frac{4 Q}{\pi \lambda^{2}}=\frac{d L_{f}}{d t}
$$


Equation (11) is combined with Eq. (10), and we obtain

$$
\begin{aligned}
v_{f}= & \frac{d L_{f}}{d t} \\
= & \frac{\alpha^{4}}{8 \mu L_{f}}\left[\frac{1}{2}\left(\lambda+2 L_{s}\right)^{2}-\frac{1}{4} \lambda^{2}\right] \\
& \times\left[\frac{4 \sigma}{\alpha \lambda}\left(\cos \theta_{\text {eq }}-\tilde{\alpha} \frac{d L_{f}}{d t} \frac{\mu}{\sigma}\right)-\rho g L_{0}\right] .
\end{aligned}
$$

$\mathrm{Yu}$ and Cheng $\frac{43}{4}$ introduced a fractal dimension that considers tortuous capillaries and then expressed the relation between $L_{f}$ and $L_{0}$ as

$$
L_{f}=\lambda^{1-D_{T}} L_{0}^{D_{T}},
$$

where $L_{f}$ refers to the length of fluid pathline, and $L_{0}$ is the height of meniscus. Velocity $v_{f}$ and linear velocity $v_{C}$ can be expressed as $\$ 4$

$$
v_{f}=\frac{d L_{f}}{d t}=D_{T} \lambda^{1-D_{T}} L_{0}^{D_{T}-1} v_{0},
$$

where $D_{T}$ refers to the fractal dimension of a tortuous capillary, $\lambda$ is the capillary diameter, and $D_{T}$ ranges from 1 to 3 . For a straight capillary, $D_{T}=1$; a high $D_{T}$ represents a tortuous and extended capillary pathline. Equations (12) and (14) are combined, and we obtain

$$
\begin{aligned}
v_{0}= & \frac{d L_{0}}{d t} \\
= & \frac{\alpha^{4}\left[\left(\lambda+2 L_{s}\right)^{2} / 2-\lambda^{2} / 4\right]}{8 \mu D_{T} \lambda^{2-2 D_{T}} L_{0}^{2 D_{T}-1}}\left[\frac { 4 \sigma } { \alpha \lambda } \left(\cos \theta_{\mathrm{eq}}\right.\right. \\
& \left.\left.-\tilde{\alpha} \frac{\mu}{\sigma} D_{T} \lambda^{1-D_{T}} L_{0}^{D_{T}-1} \frac{d L_{0}}{d t}\right)-\rho g L_{0}\right] .
\end{aligned}
$$

Equation (15) is a nonlinear implicit ordinary differential equation, and this equation cannot be typically solved. An explicit solution is thus difficult to derive. We consider two conditions to discuss this equation. Capillary force is larger than gravity or $g=0$ in a horizontal flow at the beginning of spontaneous imbibition. Therefore, Eq. (15) can be expressed as

$$
\begin{aligned}
v_{0}= & \frac{d L_{0}}{d t} \\
= & \frac{\alpha^{4}\left[\left(\lambda+2 L_{s}\right)^{2} / 2-\lambda^{2} / 4\right]}{8 \mu D_{T} \lambda^{2-2 D_{T}} L_{0}^{2 D_{T}-1}}\left[\frac { 4 \sigma } { \alpha \lambda } \left(\cos \theta_{\mathrm{eq}}\right.\right. \\
& \left.\left.-\tilde{\alpha} \frac{\mu}{\sigma} D_{T} \lambda^{1-D_{T}} L_{0}^{D_{T}-1} \frac{d L_{0}}{d t}\right)\right](g \rightarrow 0) .
\end{aligned}
$$

The method of variable separation method is used, and Eq. (16) can be expressed as

$$
\begin{gathered}
\left\{\frac{8 \mu D_{T} \lambda^{2-2 D_{T}}}{\alpha^{4}\left[\left(\lambda+2 L_{s}\right)^{2} / 2-\lambda^{2} / 4\right]} L_{0}^{2 D_{T}-1}\right. \\
\left.+\frac{4 \sigma}{\alpha \lambda} \tilde{\alpha} \frac{\mu}{\sigma} D_{T} \lambda^{1-D_{T}} L_{0}^{D_{T}-1}\right\} d L_{0} \\
=\frac{4 \sigma}{\alpha \lambda} \cos \theta_{\mathrm{eq}} d t .
\end{gathered}
$$

The imbibition length is 0 when $t=0$; thus, the initial condition is

$$
\left.L_{0}\right|_{t=0}=0 .
$$

Subsequently, with condition Eq. (18), and we can derive the final solution of spontaneous imbibition distance without gravity:

$$
\begin{aligned}
L_{0}= & \left(\left\{\frac{\alpha^{3} \sigma\left[\left(\lambda+2 L_{s}\right)^{2} / 2-\lambda^{2} / 4\right] \cos \theta_{\mathrm{eq}}}{\mu \lambda^{3-2 D_{T}} t}\right.\right. \\
& \left.+\frac{\alpha^{6} \tilde{\alpha}^{2}\left[\left(\lambda+2 L_{s}\right)^{2} / 2-\lambda^{2} / 4\right]^{2}}{4 \lambda^{4-2 D_{T}}}\right\}^{\frac{1}{2}} \\
& \left.-\frac{\alpha^{3} \tilde{\alpha}\left[\left(\lambda+2 L_{s}\right)^{2} / 2-\lambda^{2} / 4\right]}{2 \lambda^{2-D_{T}}}\right)^{\frac{1}{D_{T}}} .
\end{aligned}
$$

An explicit solution is difficult to obtain for the nonlinear ordinary differential equation (19) when gravity is nonnegligible. However, this equation may be numerically solved within an allowable error range. We use the variable-step fourth-fifth-order Runge-Kutta algorithm, which is an adaptive-step numerical analysis method for ordinary differential equations.

We perform a dimensional analysis to solve the problem for appropriate dimensionless groups to simplify the equation and enable this equation to be applicable to different scales. Based on the dimensionless method proposed by Hilpert ${ }^{42}$ and Afsharpoor and Javadpour, $\frac{36}{36}$ three independent dimensionless groups are defined as follows:

$$
L_{D}=\frac{L_{0}}{\lambda}, \quad t_{D}=\frac{t \sigma}{\mu \lambda}, \quad L_{s D}=\frac{L_{s}}{\lambda},
$$

where $L_{D}$ is the dimensionless imbibition length, $t_{D}$ is the dimensionless time, and $L_{s D}$ is the dimensionless slip length. These dimensionless groups are helpful for simplifying the equations. The dimensionless velocity is

$$
\frac{d L_{D}}{d t_{D}}=\frac{\mu}{\sigma} \frac{d L_{0}}{d t} .
$$


The dimensionless form of Eq. (16) is

$$
\begin{aligned}
v_{D}= & \frac{d L_{D}}{d t_{D}} \\
= & \frac{\alpha^{4}\left[\left(1+2 L_{s D}\right)^{2} / 2-1 / 4\right]}{8 D_{T} L_{D}^{2 D_{T}-1}}\left[\frac { 4 } { \alpha } \left(\cos \theta_{\mathrm{eq}}\right.\right. \\
& \left.\left.-\tilde{\alpha} D_{T} L_{D}^{D_{T}-1} \frac{d L_{D}}{d t_{D}}\right)-\frac{\rho g \lambda^{2} L_{D}}{\sigma}\right]
\end{aligned}
$$

with initial conditions $\left.L_{D}\right|_{t_{D}=0}=0$.

When $\frac{4 \sigma \cos \theta}{\alpha \lambda}>>\rho \lambda L_{D}, g \rightarrow 0$, the dimensionless solution is

$$
\begin{aligned}
L_{D}= & \left(\left\{\alpha^{3}\left[\frac{\left(1+2 L_{s D}\right)^{2}}{2}-\frac{1}{4}\right] \cos \theta_{\mathrm{eq}} t\right.\right. \\
& \left.+\frac{\alpha^{6} \tilde{\alpha}^{2}\left[\left(1+2 L_{s D}\right)^{2} / 2-1 / 4\right]^{2}}{4}\right\}^{\frac{1}{2}} \\
& \left.-\frac{\alpha^{3} \tilde{\alpha}\left[\left(1+2 L_{s D}\right)^{2} / 2-1 / 4\right]}{2}\right)^{\frac{1}{D_{T}}} .
\end{aligned}
$$

We use the variable-step fourth-fifth-order Runge-Kutta algorithm when $g$ is considered to obtain the numerical solution of the nonlinear ordinary differential equation expressed as

$$
\begin{aligned}
v_{0}= & \frac{d L_{0}}{d t}=\frac{\alpha^{4} \pi}{32 \mu \lambda^{1-D_{T}} L_{0}^{D_{T}}\left(D_{T} L_{0}^{D_{T}-1} \lambda^{1-D_{T}}\right)} \\
& \times\left[\frac{1}{2}\left(\lambda+2 L_{s}\right)^{2}-\frac{1}{4} \lambda^{2}\right]\left(\frac{4 \sigma \cos \theta}{\alpha \lambda}-\rho g L_{0}\right) .
\end{aligned}
$$

The accumulated volume of the liquid is imbibed into the capillary, and the imbibition speed can be derived by integrating the solution of $L_{0}(t)$ into Eq. (24):

$$
m=\frac{1}{4} \rho \pi \lambda^{2} L_{f}, \quad q=\frac{d m}{d t}=\frac{1}{4} \rho \pi \lambda^{2} \frac{d L_{f}}{d t} .
$$

Similarly, we can generate dimensional and dimensionless equations for the accumulated weight of liquid and imbibition speed based on the solution of Eq. (23).

\subsection{Spontaneous Imbibition in Porous Media}

Pore space is more complicated than a single capillary in porous media. Capillary bundle model is an effective and classic means of investigating the flow in porous media. In this model, the porous media are considered a bundle of capillary tubes of the same length, and the spaces among the tubes are sealed by a cementing material. Porous media are considered tortuous capillaries with variableshaped apertures in this study. The pore size distribution of capillaries significantly influences the spontaneous imbibition process. The pore size distribution of porous media, as a capillary bundle model, is regarded the same as the pore size distribution of capillaries. In addition to idealized porous media, lognormal distribution function is introduced. This function is demonstrated as a favorable means of representing the pore size distribution of porous media, $\stackrel{45 \mid 46}{4}$ thereby denoting that pore space exhibits a generalized the lognormal distribution as

$$
f(r)=\frac{1}{\sqrt{2 \pi} \ln \sigma} \exp \left[-\left(\frac{\ln \left(r^{\prime} / \bar{r}^{\prime}\right)}{\sqrt{2} \ln \sigma}\right)^{2}\right],
$$

where $r^{\prime}=\frac{\left(r-r_{\min }\right)\left(r_{\max }-r_{\min }\right)}{r_{\max }-r}$, and $r$ is the equivalent radius of pores. $\sigma$ and $\bar{r}^{\prime}$ are the distribution parameters that characterize the distribution properties of $r^{\prime} \cdot \bar{r}^{\prime}$ refers to the mean or expectation of the distribution, and sigma $\sigma$ is the standard deviation. $f(r)$ is the percent volume of voids in radius $r$. The cumulative distribution function can be expressed as

$$
\begin{aligned}
F(r)= & \frac{1}{\sqrt{2 \pi} \ln \sigma} \int_{r}^{r_{\max }} \exp \left[-\left(\frac{\left.\ln r^{\prime}(x)-\ln \bar{r}^{\prime}\right)}{\sqrt{2} \ln \sigma}\right)^{2}\right] \\
& \times \frac{\left(r_{\max }-r_{\min }\right)}{\left(r_{\max }-x\right)\left(x-r_{\min }\right)} d x
\end{aligned}
$$

where $F(r)$ is the percent volume of voids in radius larger than $r$. Diamond et al. indicated that pores concentrate on the mean or expectation of the distribution $\bar{r}^{\prime}$ when $\sigma$ decreases, and the curve peak is high, as depicted in Fig. 2 .

For a real shale or tight sample, pore size distribution is derived from numerous methods, such as mercury injection capillary pressure (MICP), lowfield nuclear magnetic resonance (NMR), and $\mathrm{N}_{2}$ adsorption. Percentages of various pore shapes can be derived from focused ion beam (FIB)/scanning electron microscope (SEM) image analysis. Among the methods, the MICP is relatively fast and convenient. Hinai et al. $\frac{47}{a}$ confirmed that the MICP method can be a reliable technique for understanding pore throat size distribution decrease to $3 \mathrm{~nm}$ and determining most porosity involved in fluid transportation. The method is also reliable in shale samples. The $\mathrm{N}_{2}$ adsorption method can disclose pore size information in the micropore range. This method is accurate in measuring nanopores smaller 


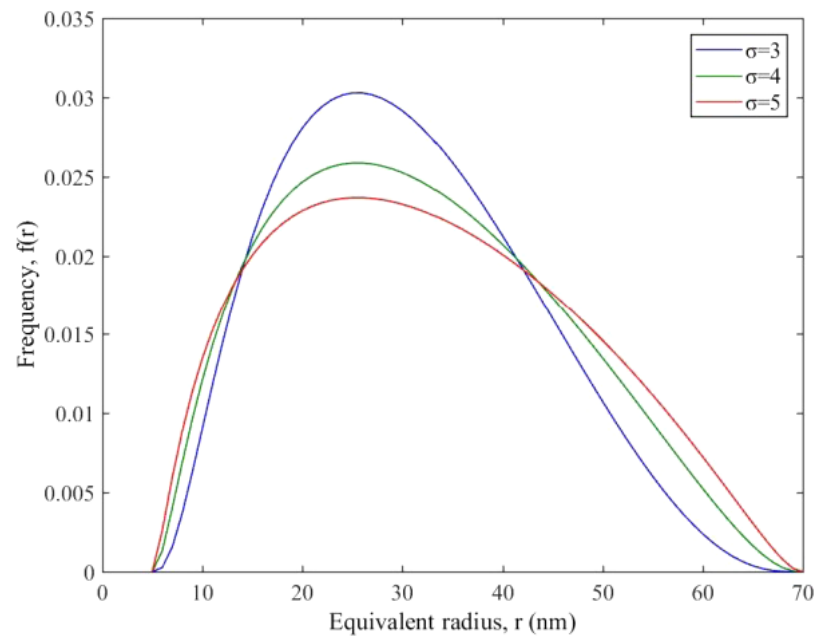

(a)

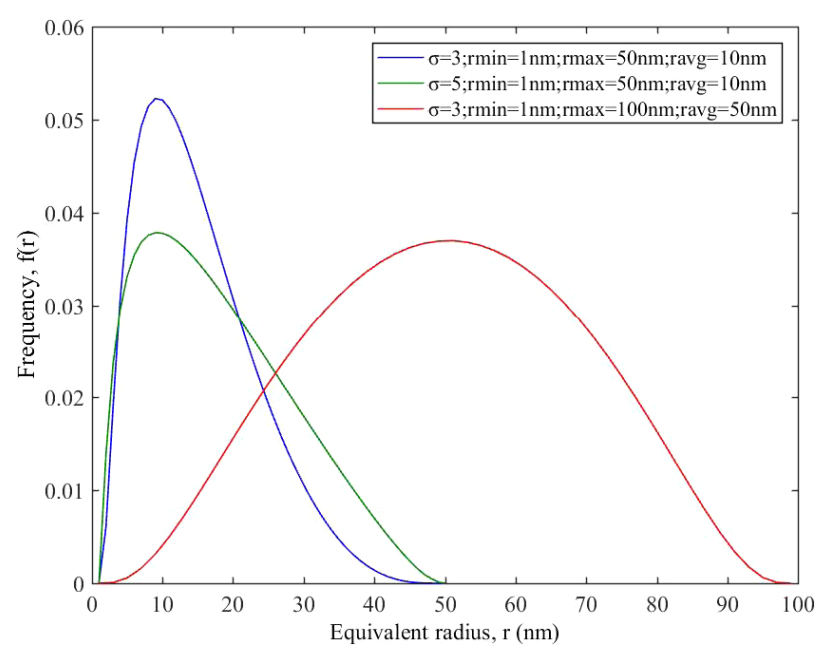

(b)

Fig. 2 Schematic of the effects of standard deviation $\sigma$ and pore range on distribution function.

than $2 \mathrm{~nm}$. The low-field NMR is a nondestructive method for examining the pore size information after full saturation by water. This method can also distinguish organic and inorganic pores by saturating different kinds of fluids $\stackrel{48}{50}$ The NMR measures connected and unconnected pores at all scales; thus, the porosity is commonly larger from the NMR than from the MICP and $\mathrm{N}_{2}$ adsorption. 51

The spontaneous imbibition equation, which considers the slip effect in nanopores and dynamic contact angle, can be expressed as

$$
\begin{aligned}
m & =\sum_{r=r_{\text {min }}}^{r_{\text {max }}} f(r) A_{r} L_{f}(r), \\
q & =\sum \frac{\Delta m}{\Delta t}=\rho \sum_{r=r_{\text {min }}}^{r_{\max }} f(r) A_{r} v(r) .
\end{aligned}
$$

The average imbibition height can be expressed as

$$
\overline{L_{D}}(t)=\frac{\sum_{r=r_{\min }}^{r_{\max }} L_{D}(r, t) f(r) A_{r}}{\sum_{r=r_{\min }}^{r_{\max }} f(r) A_{r}} .
$$

\subsection{Fractal Characteristics of Porous Media}

Pore spaces in cores exhibit fractal properties in a specific scale,, 52 57 thereby suggesting that such spaces can be rebuilt as fractal models based on their self-imitating properties. The fractal models provide an efficient means of expressing the pore size distribution to calculate permeability. Many permeability models have been developed based

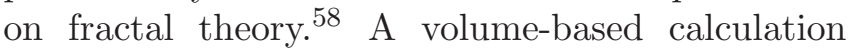
method of fractal dimension value is introduced to evaluate the influence of fractal dimension from the pore size distribution on the spontaneous imbibition process. Many calculation methods have been established. Pfeifer and Avnir ${ }^{59}$ developed the first approach in 1983. The fundamental equation is expressed as

$$
-\frac{d V}{d r} \propto r^{2-D_{f}},
$$

where $V$ is the volume of pores at radius $r$. $D_{f}$ is the volume fractal dimension, which indicates that the volume of pores in the porous media can be processed in fractal terms with the development of a scaling procedure. This approach has been approved by several works $\underline{60}$ and is estimated by transferring the pore size distribution data in a bilogarithmic diagram and calculating the slope of straight line as follows:

$$
\lg \left(-\frac{d V}{d r}\right)=m \lg r+q .
$$

The relationship between gradients $m$ and fractal dimension $D_{f}$ is $D_{f}=2-m$. $D_{f}$ is commonly between 2 and 3 . A high $D_{f}$ indicates a high complexity of the micro-nanostructure. Generally, $q$ increases with the total porosity. Under some circumstances, only one part of pore size distribution data follows the linear relationship, that is, only certain parts of the micro-nanostructures possess self-like properties.

\section{MODEL VERIFICATION}

Previously published datasets are used to validate the constructed model ${ }^{23 / 42}$ The published data and model results under similar working conditions are drawn under the same coordinates. The imbibition 


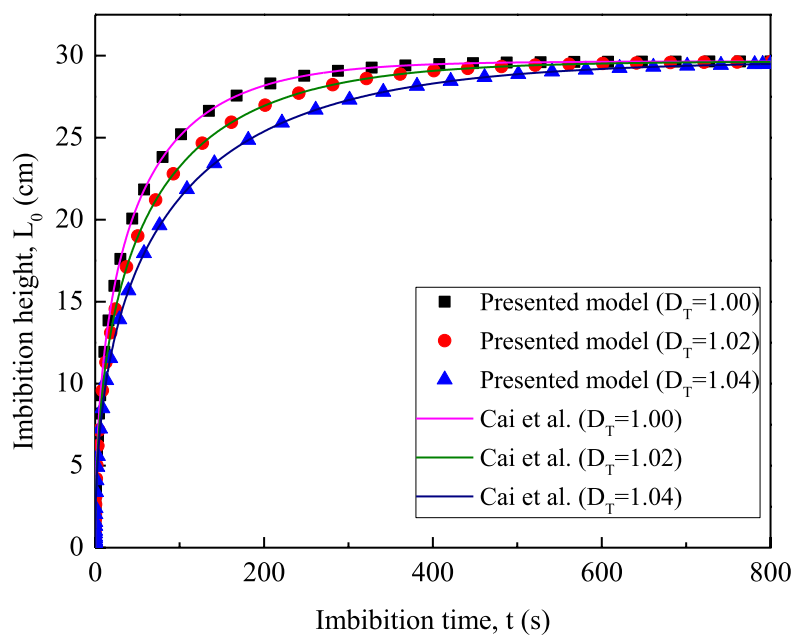

(a) Model verification between $L_{0}$ and $t$

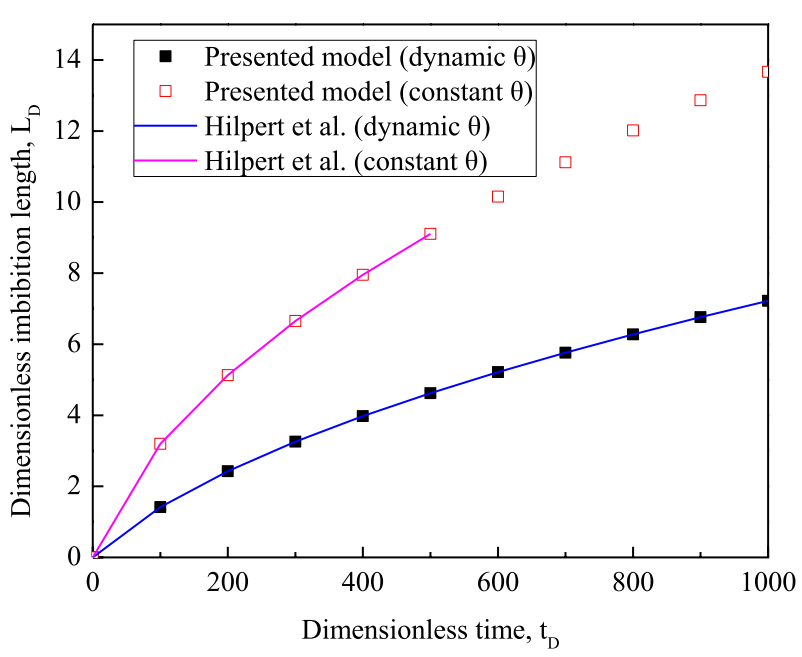

(c) Model verification between $L_{D}$ and $t_{D}$

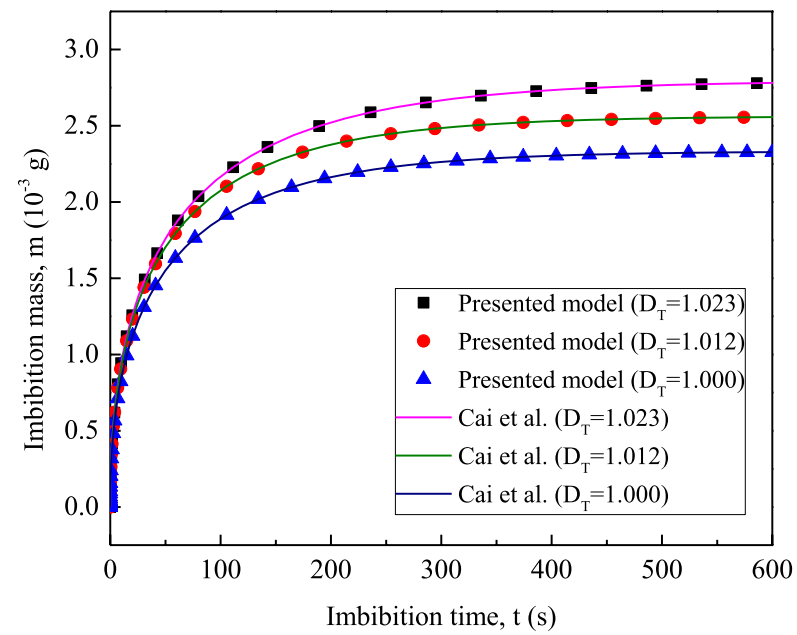

(b) Model verification between $m$ and $t$

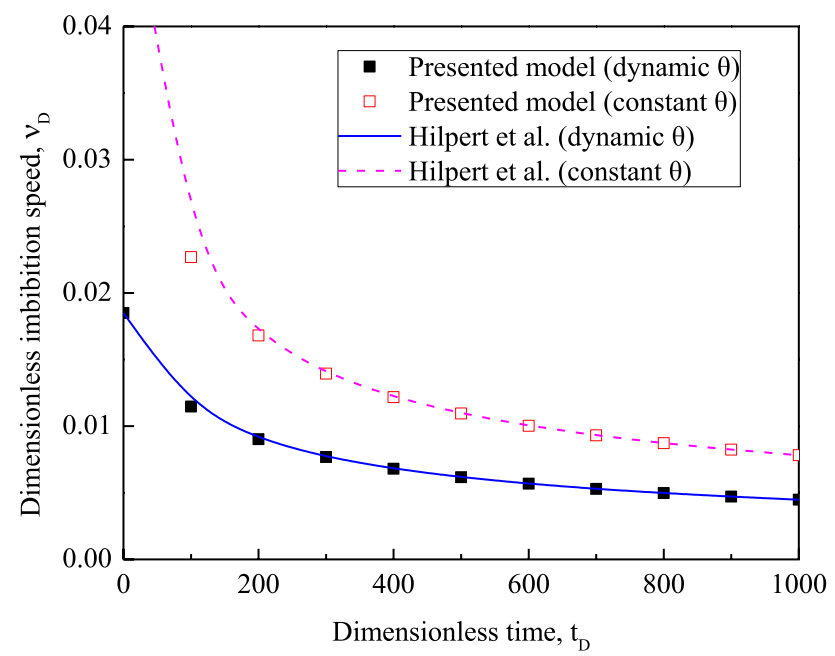

(d) Model verification between $V_{D}$ and $t_{D}$

Fig. 3 Data of model verification.

height, imbibition mass, dimensionless height, and dimensionless velocity in both cases are verified. The verification results are displayed in Fig. 3 .

Many physical properties of capillary influence imbibition height. Cai et al. ${ }^{23}$ derived the equations of imbibition height and accumulated imbibition weight versus time in a tortuous circular capillary. The model of Cai can be considered a special simplified condition of the presented model by disregarding liquid slip, noncircular cross-section influence, and dynamic contact angle. Equation (24) can be simplified as the model of by setting $L_{s}=0 \cos \theta_{\text {eq }}=$ $1 \tilde{\alpha}=1$ and $\alpha=1$. The numerical solution through ODE45 method is derived by taking the condition parameters of $\sigma=72.5 \mathrm{mN} / \mathrm{m}, \rho=0.998 \mathrm{~g} / \mathrm{cm}^{3}$, $\mu=1 \mathrm{mPas}$, and $\theta_{\mathrm{eq}}=1$. The results are the dots in Figs. 3a and 3b. As previously discussed, the fractal dimension is large. Tortuosity is responsible for reducing imbibition rate. Considerable time is required to reach the equilibrium height in tortuous tubes. However, fractal dimension or tortuosity has no influence on equilibrium height. Tortuous tubes have extended pathlines at the same height, thereby indicating a high imbibition mass. The result shows that our model significantly coordinates with the published data.

Numerical solutions of spontaneous imbibition that considers dynamic contact angle have been verified. Hilpert derived semi-analytical solutions of the dynamic contact angle on liquid infiltration into capillaries in horizontal, upward, and downward directions. Downward solutions include a steady-state, accelerating, and decelerating process. The studies of Hilpert mainly focused on force 
displacement in a circular capillary without any slip effects. Our numerical solution through the ODE45 method by setting $L_{s}=0, D_{T}=1$, and $\alpha=1$ can be compared with the semi-analytical solution according to the inputting data of calculation, namely, $\tilde{\alpha}=18.5$, proposed by Hilpert and static contact angle $\theta_{\text {eq }}=70^{\circ}$. If the contact angle remains stable in the imbibition process $\tilde{\alpha}=0$, then the dynamic contact angle $\theta$ is equal to the static contact angle, that is, $\theta=\theta_{\text {eq }}=70^{\circ}$. The influence of the dynamic contact angle on dimensionless height and velocity is presented in Figs. 3 . and $3 \mathrm{~d}$. The lines are the results of Hilpert, and the dots are our solutions. Solid lines indicate the contact angle changes in the imbibition process, while dashed lines are the ignored contact angle changes. The result verifies that our model coordinates well with the published data.

Dimensionless height is defined as $L_{D}=L_{0} / \lambda$ in the present study and $L_{D}=L_{0} / R$ or $L_{D}=2 L_{0} / \lambda$ in the work of Hilpert. Therefore, the data of original dimensionless height by Hilpert are converted.

\section{RESULT AND DISCUSSION}

\subsection{Dimensionless Slip Length}

In Fig. 4, the spontaneous imbibition curve varies with different dimensionless slip lengths. The difference increases with an extended dimensionless slip length, thereby indicating that slip length should be considered in the research of the imbibition process. Figure 4 also demonstrates that the average dimensionless imbibition height decreases when the slip length is extended. Slip effect is more obvious in the micro-nanopores of shales than in sandstones. The imbibition speed is doubled when the slip length reaches nearly the same as the capillary diameter. Therefore, slip length can easily reach the magnitude, even exceed pore diameter, in micro-nanopores. The neglect of the slip effect will underestimate the imbibition speed in shale or tight samples.

The $\log -\log$ curve presents that $\overline{L_{D}}$ decreases at the beginning of the imbibition process. This decrease is caused by the ODE45 method. Iteration is difficult to converge when the iterative solution method begins. The solutions become highly reliable with the increase in the number of iterative time. An obvious linear part emerges in the middle of the curve. The slope is called imbibition index according to spontaneous imbibition theory.

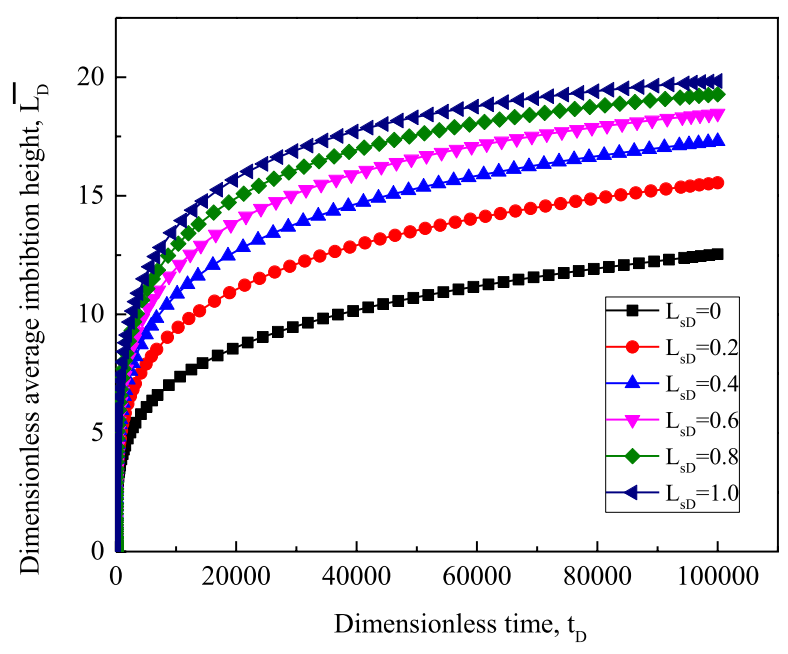

(a)

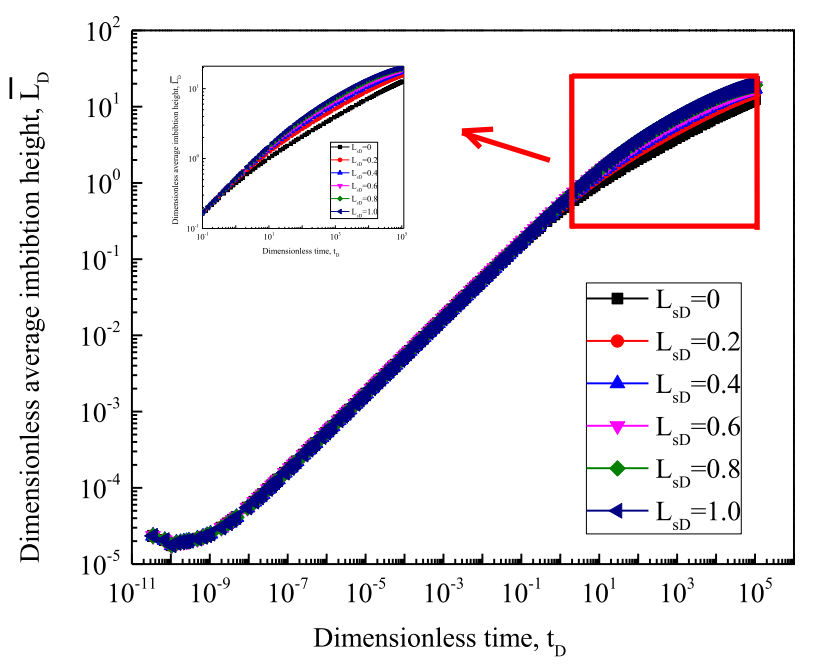

(b)

Fig. 4 Influence of dimensionless slip length on the spontaneous imbibition process.

In sandstones, $n \approx 0.5$. In our model, the $n$ in linear part is $1 / 2 D_{T}$. The influence of gravity emerges while meniscus rises, thereby causing the curve to go downward. The partially enlarged image denotes that the short dimensionless slip length rapidly deviates from the linear part.

\subsection{Shape Factor}

Figure 5 illustrates the spontaneous imbibition curves with various shape factors. The definition and expression of shape factors by using Eq. (3) imply that $\alpha$ is larger in an equilateral triangle than in a square. A circle has the smallest $\alpha$. The spontaneous imbibition characteristics of the different shapes with the same hydraulic diameter 


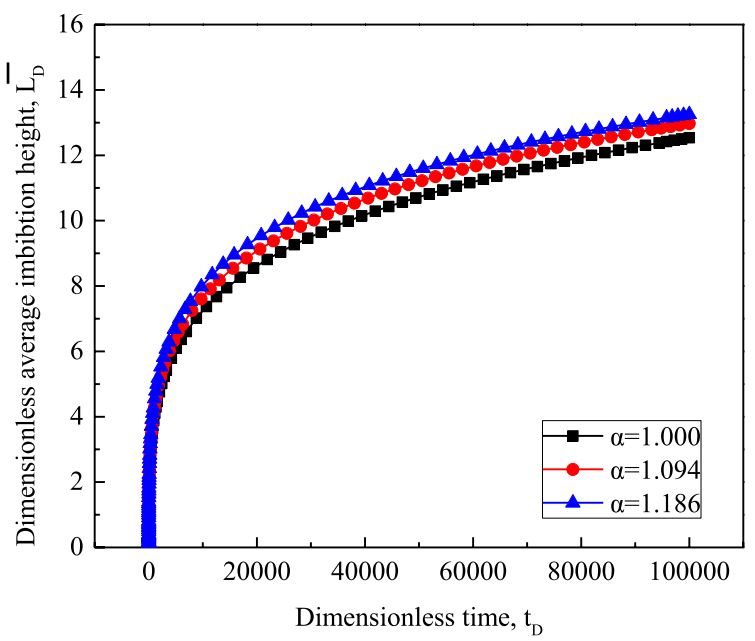

(a)

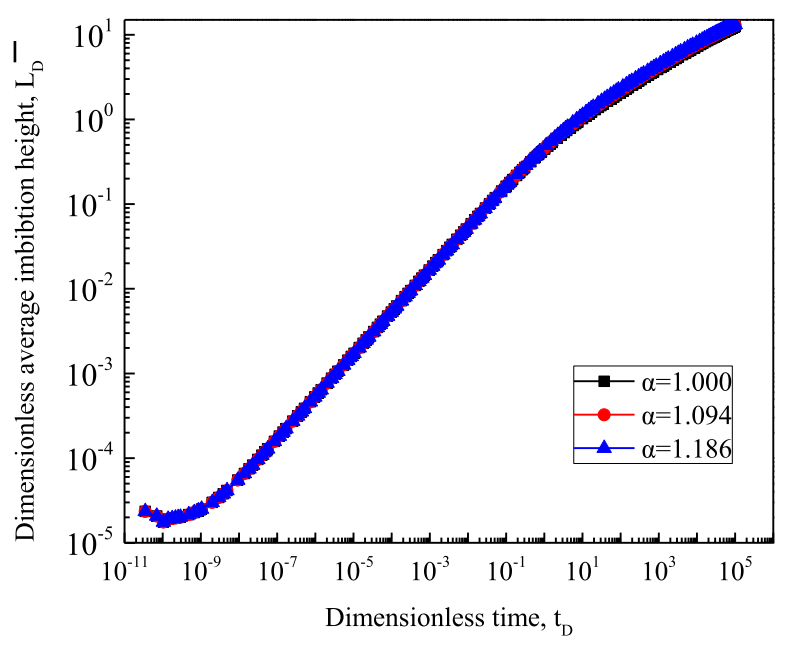

(b)

Fig. 5 Influence of dimensionless slip length on the spontaneous imbibition process.

are discussed. The simulation result of the spontaneous imbibition indicates that triangle tubes have a faster imbibition speed than the other shapes or consumes less time to reach the same height. Circular tubes are the slowest, considering the slip effect and dynamic contact angle. The hydraulic diameter is considered an equivalent diameter for all tubes in our model. The cross-sectional area is larger in a square than in a circle given the same hydraulic diameter, that is, $S_{\text {square }}-S_{\text {circle }}=D^{2}-\frac{\pi}{4} D^{2}>0$. The wetted perimeter is also larger in a square than in a circle, that is, $A_{\text {square }}-A_{\text {circle }}=4 D-\pi D>0$. Flowing resistance is minimal in a large crosssectional area. A large wetted perimeter leads to a high capillary force.

The $\log -\log$ curve presents that $\overline{L_{D}}$ decreases because the ODE45 method does not converge at

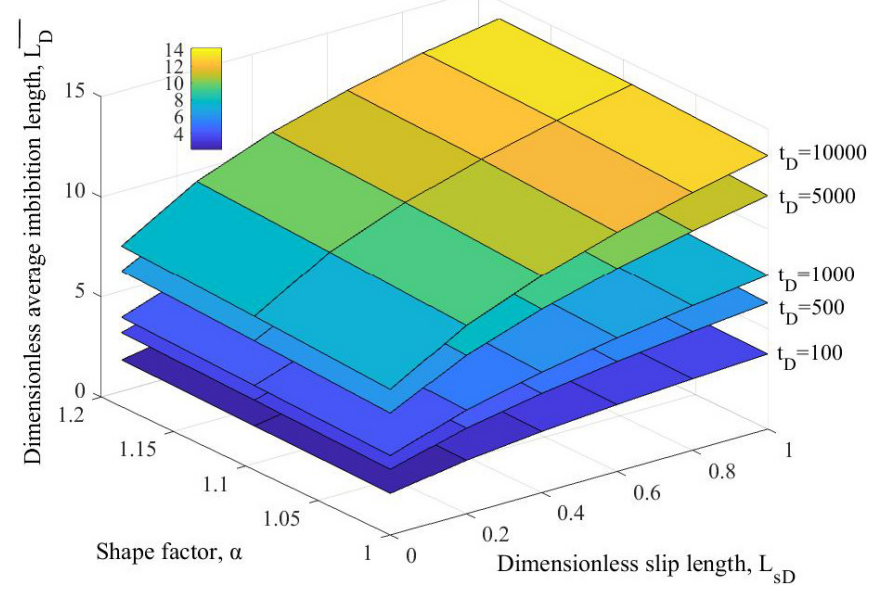

(a)

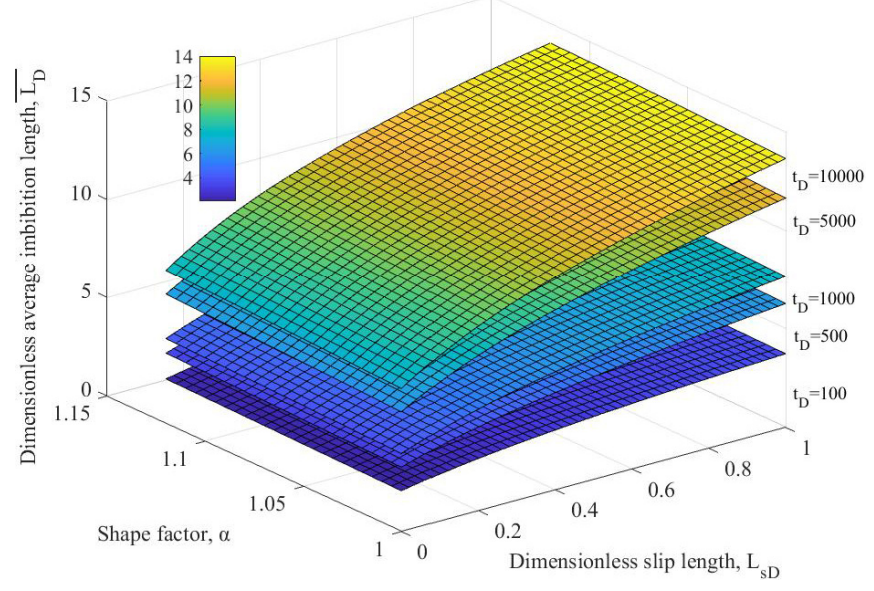

(b)

Fig. 6 3D parameter table of the spontaneous imbibition process.

the beginning of the numerical solving process. A linear slope also occurs. The slope bends down from the linear relationship when the influence of gravity increases.

Empirically generalized imbibition equations that consider the slip effects of liquid and dynamic contact angle are developed in this study. Analytic solutions at $g=0$ and numerical solution at $g \neq 0$ are derived.

Slip length, shape factor, and imbibition speed influence an average imbibition length. We create a 3D parameter figure (Fig. 6) to visualize the influences of these parameters. In Fig. 67, a warm color denotes a high $\overline{L_{D}}$. Each plane indicates the same dimensionless imbibition time. Figure 60 p provides the solution after a spline interpolation. Figure 6 depicts that the increase in $\overline{L_{D}}$ speed becomes slow 
when the imbibition time increases. The time interval between the top two layers is 5000, whereas that of the bottom two layers is only 400. A high shape factor and dimensionless slip length gain a high imbibition speed. Figure is helpful for understanding the parameter that mostly influences $\overline{L_{D}}$.

\section{CONCLUSION}

The liquid slip effect in spontaneous imbibition is investigated. An empirically generalized spontaneous imbibition equation that considers the liquid slip effects and dynamic contact angle is developed. An analytic solution at $g=0$ and numerical solution at $g \neq 0$ are derived, and the model solution is verified by using published data.

The spontaneous imbibition model that considers the slip boundary indicates that the imbibition speed can be twice of the speed in which a slip boundary condition is disregarded when nondimensionless slip length reaches 1 . The liquid slip effect is important in investigating the liquid flow and imbibition process in the micro-nanopores of shale. The neglect of slip effect will underestimate the imbibition speed in shale samples. Shape factor also influences the imbibition process; a large shape factor gains a high imbibition length simultaneously with the same equivalent diameter.

A 3D parameter chart is created to clarify the influences of parameters. The increase in the speed of $\overline{L_{D}}$ becomes slow while the imbibition time increases. A high shape factor and dimensionless slip length gain a high imbibition speed. The chart is helpful for understanding the parameter that mostly influences $\overline{L_{D}}$. This study has a certain room for improvement in the research of slippage of imbibition in complex pore networks. However, this study provides a favorable foundation for future works.

\section{ACKNOWLEDGMENTS}

This work was supported by Research Foundation of China University of Petroleum-Beijing At Karamay (RCYJ2016B-01-001) and supported by the National Natural Science Foundation of China (Grants 51604287, 51490652).

\section{REFERENCES}

1. S. A. Holditch, Tight gas sands, SPE J. Petrol. Technol. 58 (2006) 86-93.
2. C. R. Clarkson, J. L. Jensen and T. Blasingame, Reservoir engineering for unconventional reservoirs: What do we have to consider, in North American Unconventional Gas Conf. and Exhibition (Society of Petroleum Engineers, 2011), pp. 1-45.

3. M. Y. Soliman, J. Daal and L. East, Fracturing unconventional formations to enhance productivity, J. Nat. Gas Sci. Eng. 8 (2012) 52-67.

4. L. Yang et al., Imbibition inducing tensile fractures and its influence on in-situ stress analyses: A case study of shale gas drilling, J. Nat. Gas Sci. Eng. 26 (2015) 927-939.

5. Z. Ye, A. Ghassemi and S. Riley, Fracture properties characterization of shale rocks, in Unconventional Resources Technology Conf. (Unconventional Resources Technology Conference, 2016), pp. 1-13.

6. Z. Ye et al., Laboratory investigation of fluid flow and permeability evolution through shale fractures, in Unconventional Resources Technology Conf. (Unconventional Resources Technology Conference, 2017), pp. 1-15.

7. D. B. Bennion and F. B. Thomas, Formation damage issues impacting the productivity of low permeability, low initial water saturation gas producing formations, J. Energy Resour. Technol. 127(3) (2005) 240-247.

8. F. Civan, Reservoir Formation Damage (Gulf Professional Publishing, US, 2011).

9. D. Zhang et al., Influence of water saturation on the mechanical behaviour of low-permeability reservoir rocks, Energies 10(2) (2017) 236.

10. A. Javaheri, H. Dehghanpour and J. M. Wood, Tight rock wettability and its relationship to other petrophysical properties: A Montney case study, J. Earth Sci. 28(2) (207) 381-390.

11. M. J. Yun et al., Fractal analysis of power-law fluid in a single capillary, Chin. Phys. Lett. 25(2) (2008) 616-619.

12. Y. Shen et al., Water imbibition of shale and its potential influence on shale gas recovery - A comparative study of marine and continental shale formations, J. Nat. Gas Sci. Eng. 35 (2016) 1121-1128.

13. Y. Shen et al., Effect of water imbibition on shale permeability and its influence on gas production, Energy Fuels 31(5) (2017) 4973-4980.

14. K. Shanley, R. Cluff and J. Robinson, Factors controlling prolific gas production from lowpermeability sandstone reservoirs: Implications for resource assessment, prospect development, and risk analysis, AAPG Bull. 88(8) (2004) 1083-1121.

15. X. Cui, A. Bustin and R. M. Bustin, Measurements of gas permeability and diffusivity of tight reservoir rocks: Different approaches and their applications, Geofluids 9 (2009) 208-223.

16. B. Yuan and R. G. Moghanloo, Analytical evaluation of nanoparticles application to reduce fines 
migration in porous media, SPE J. 21(6) (2016) 2317-2332.

17. A. Bertoncello et al., Imbibition and water blockage in unconventional reservoirs: Well management implications during flowback and early production, in SPE/EAGE European Unconventional Resources Conf. Exhibition (Society of Petroleum Engineers, 2014), pp. 1-10.

18. J. C. Cai et al., Generalized modeling of spontaneous imbibition based on Hagene Poiseuille flow in tortuous capillaries with variably shaped apertures, Langmuir 30(18) (2014) 5142-5151.

19. P. Fakcharoenphol et al., The effect of osmotic pressure on improve oil recovery from fractured shale formations, in SPE Unconventional Resources Conf. (Society of Petroleum Engineers, 2014), pp. 1-12.

20. Y. Shen et al., Imbibition characteristic of shale gas formation and water-block removal capability, Sci. Sin. Phys. Mech. Astron. 47(11) (2017) 114609.

21. Q. B. Meng, H.Q. Liu and W. Jing, A critical review on fundamental mechanisms of spontaneous imbibition and the impact of boundary condition, fluid viscosity and wettability, Adv. Geo-Energy Res. 1(1) (2017) 1-17.

22. J. C. Cai and B. M. Yu, Advances in studies of spontaneous imbibition in porous media, Adv. Mech. 42(6) (2012) 735-754.

23. J. C. Cai et al., Capillary rise in a single tortuous capillary, Chin. Phys. Lett. 27(5) (2010) 054701.

24. J. C. Cai and B. M. Yu, A discussion of the effect of tortuosity on the capillary imbibition in porous media, Transp. Porous Media 89(2) (2011) 251-263.

25. D. Lin et al., Review on gas flow and recovery in unconventional porous rocks, Adv. Geo-Energy Res. 1(1) (2017) 39-53.

26. C. Li et al., Analysis of spontaneous imbibition in fractal tree-like network system, Fractals 24 (2016) 1650035 .

27. C. Li et al., Analysis of capillary rise in asymmetric branch-like capillary, Fractals 24 (2016) 1650024.

28. R. Liu et al., Recent developments on relationships between the equivalent permeability and fractal dimension of two-dimensional rock fracture networks, J. Nat. Gas Sci. Eng. 45 (2017) 771-785.

29. W. Wei et al., A numerical study on fractal dimensions of current streamlines in two-dimensional and three-dimensional pore fractal models of porous media, Fractals 23(1) (2015) 1540012.

30. X. Guo, Y. Shen and S. He, Quantitative pore characterization and the relationship between pore distributions and organic matter in shale based on Nano-CT image analysis: A case study for a lacustrine shale reservoir in the Triassic Chang 7 member, Ordos Basin, China, J. Nat. Gas Sci. Eng. 27 (2015) 1630-1640.
31. B. Yuan et al., A new analytical multi-linear solution for gas flow toward fractured horizontal wells with different fracture intensity, J. Nat. Gas Sci. Eng. 23 (2015) 227-238.

32. H. Liu et al., Some key technical issues in modelling of gas transport process in shales: A review, Geomech. Geophys. Geo-Energy Geo-Resour. 4(2) (2016) 231-243.

33. F. Javadpour, D. Fisher and M. Unsworth, Nanoscale gas flow in shale sediments, J. Canad. Petrol. Technol. 46 (2007) 55-61.

34. F. Javadpour, Nanopores and apparent permeability of gas flow in mudrocks (shales and siltstone), J. Canad. Petrol. Technol. 48 (2009) 16-21.

35. F. Javadpour, M. McClure and M. Naraghi, Slipcorrected liquid permeability and its effect on hydraulic fracturing in shale, Fuel 160 (2015) 549559 .

36. A. Afsharpoor and F. Javadpour, Liquid slip flow in a network of shale noncircular nanopores, Fuel 180 (2016) 580-590.

37. R. Liu et al., Estimating permeability of porous media based on modified Hagen-Poiseuille flow in tortuous capillaries with variable lengths, Microfluid. Nanofluid. 20(8) (2016) 120.

38. J. C. Cai et al., Generalized modeling of spontaneous imbibition based on Hagen-Poiseuille flow in tortuous capillaries with variably shaped apertures, Langmuir 30(18) (2014) 5142-5151.

39. M. Hilpert, Effects of dynamic contact angle on liquid infiltration into horizontal capillary tubes: (Semi)-analytical solutions, J. Colloid Interf. Sci. 337(1) (2009) 131-137.

40. E. Schäffer and P. Wong, Dynamics of contact line pinning in capillary rise and fall, Phys. Rev. Let. 80(14) (1998) 3069-3072.

41. M. Popescu, J. Ralston and R. Sedev, Capillary rise with velocity-dependent dynamic contact angle, Langmuir 24(21) (2008) 12710-12716.

42. M. Hilpert, Liquid withdrawal from capillary tubes: Explicit and implicit analytical solution for constant and dynamic contact angle, J. Colloid Interf. Sci. 351(1) (2010) 267-276.

43. B. M. Yu and P. Cheng, A fractal permeability model for bi-dispersed porous media, Int. J. Heat Mass Transf. 45(14) (2002) 2983-2993.

44. J. C. Cai et al., Fractal characterization of spontaneous concurrent imbibition in porous media, Energy Fuels 24(3) (2010) 1860-1867.

45. S. Diamond and W. L. Dolch, Generalized lognormal distribution of pore sizes in hydrated cement paste, J. Colloid Interf. Sci. 38(1) (1972) 234-244.

46. S. I. Hwang and S. E. Powers, Using particle-size distribution models to estimate soil hydraulic properties, Soil Sci. Soc. Am. J. 67 (2003) 1103-1112. 
47. A. Hinai et al., Comparisons of pore size distribution: A case from the Western Australian gas shale formations, J. Unconv. Oil Gas Res. 8 (2014) 1-13.

48. J. Li et al., Four-pore modeling and its quantitative logging description of shale gas reservoir, Oil Gas Geol. 35(2) (2014) 266-271 (in Chinese).

49. M. Meng et al., Monitor the process of shale spontaneous imbibition in co-current and counter-current displacing gas by using low field nuclear magnetic resonance method, J. Nat. Gas Sci. Eng. 27 (2015) 336-345.

50. M. Meng et al., Investigation on the variation of shale permeability with spontaneous imbibition time: Sandstones and volcanic rocks as comparative study, J. Nat. Gas Sci. Eng. 27 (2015) 1546-1554.

51. J. Li, W. Jin and L. Wang, Quantitative evaluation of organic and inorganic pore size distribution by NMR: A case from the Silurian Longmaxi formation gas shale in Fuling area, Sichuan Basin, Oil Gas Geol. 37(1) (2016) 129-134.

52. A. H. Thompson, A. J. Katz and C. E. Krohn, The microgeometry and transport properties of sedimentary rocks, Adv. Phys. 36(5) (1987) 625-694.

53. P. M. Adler, Transports in fractal porous media, J. Hydrol. 187(1-2) (1996) 195-213.
54. P. M. Adler and J. F. Thovert, Real porous media: Local geometry and macroscopic properties, Appl. Mech. Rev. 51(9) (1998) 537-585.

55. J. F. Thovert, F. Wary and P. M. Adler, Thermal conductivity of random media and regular fractals, J. Appl. Phys. 68(8) (1990) 3872-3883.

56. B. M. Yu, Fractal character for tortuous streamtubes in porous media, Chin. Phys. Lett. 22(1) (2005) 158160.

57. B. M. Yu and J. H. Li, Some fractal characters of porous media, Fractals 9(3) (2001) 365-372.

58. J. C. Cai et al., Recent advances on fractal modeling of permeability for fibrous porous media, Fractals 23(1) (2015) 1540006.

59. P. Pfeifer and D. Avnir, Chemistry in noninteger dimensions between two and three. I. Fractal theory of heterogeneous surfaces, J. Chem. Phys. 79(7) (1983) 3558-3565.

60. C. Atzeni, G. Pia and U. Sanna, Fractal modelling of medium-high porosity SiC ceramics, J. Eur. Ceram. Soc. 28(14) (2008) 2809-2814.

61. C. Atzeni, G. Pia and U. Sanna, A geometrical fractal model for the porosity and permeability of hydraulic cement pastes, Constr. Build. Mater. 24(10) (2010) 1843-1847. 